\title{
Effects of taxol resistance gene 1 on the cisplatin response in gastric cancer
}

\author{
SHUQUAN DUAN, JIE YIN, ZHIGANG BAI and ZHONGTAO ZHANG \\ Department of General Surgery, Beijing Friendship Hospital, Capital Medical University, Beijing Key Laboratory of \\ Cancer Invasion and Metastasis Research and National Clinical Research Center for \\ Digestive Diseases, Beijing 100050, P.R. China
}

Received August 15, 2017; Accepted February 12, 2018

DOI: $10.3892 / 01.2018 .8390$

\begin{abstract}
Cisplatin is used to treat multiple types of solid tumor, including gastric cancer. Although cisplatin initially exhibits good efficacy, therapeutic failure often occurs owing to the development of chemoresistance. To the best of our knowledge, the underlying mechanism of cisplatin resistance remains unknown. The aim of the present study was to assess whether taxol resistance gene 1 (TXR1) has a role in cisplatin response in gastric cancer. The expression of TXR1 in fresh-frozen tissues of patients with gastric cancer who were sensitive or resistance to cisplatin was assessed. The level of TXR1 expression was significantly higher in cisplatin-resistant specimens than that in cisplatin-sensitive specimens. Next, the gastric cancer SGC-7901 cell line was exposed to cisplatin to establish a cisplatin-resistance subline, termed SGC-7901/DDP, which exhibited a 6-fold increases in the level of resistance. TXR1 expression was elevated in SGC-7901/DDP cells. Overexpression of TXR1 induced cisplatin resistance in SGC-7901 cells. Downregulation of TXR1 reversed the drug resistance caused by elevation of TXR1 expression in SGC-7901/DDP cells. Animal experiments proved the effect of TXR1 in inducing cisplatin resistance in vivo. Further investigation revealed that TXR1 regulated cisplatin resistance via apoptosis. In conclusion, TXR1 is worthy of further in-depth study as a potential therapeutic target in patients with gastric cancer.
\end{abstract}

Correspondence to: Dr Zhongtao Zhang or Dr Zhigang Bai, Department of General Surgery, Beijing Friendship Hospital, Capital Medical University, Beijing Key Laboratory of Cancer Invasion and Metastasis Research and National Clinical Research Center for Digestive Diseases, 95 Yongan Road, Beijing 100050, P.R. China E-mail: zhangzht@medmail.com.cn

E-mail: bai_zhg@163.com

Key words: cisplatin, chemoresistance, taxol resistance gene 1, gastric cancer, apoptosis

\section{Introduction}

Gastric cancer is a malignancy with a multi-factorial etiology (1). Owing to a lack of effective screening methods, the majority of patients are diagnosed at an advanced disease stage. For these patients, systemic chemotherapy is the main treatment method (2). Cisplatin is one of the most potent antitumor agents and displays a good curative effect against a wide variety of solid tumor types, including gastric cancer (3). Cisplatin exerts a cytotoxic effect by interacting with DNA to form DNA adducts, which can activate a number of downstream signal transduction pathways, and eventually leads to activation of apoptosis (4). Unfortunately, the majority of gastric cancer patients fail to respond to chemotherapy owing to the development of drug resistance (5). Therefore, the development of methods to overcome drug resistance and improve clinical treatment efficacy is required.

Recent studies have shown that taxol resistance protein 1 (TXR1), which was first identified as a taxol resistance-associated gene in prostate cancer, was differently expressed in a number of tumor types, including gastric cancer (6), breast cancer (7) and non-small cell lung cancer (8). It has been reported that high levels of TXR1 mRNA expression resulted in the development of resistance to taxol in $\mathrm{HeLa}$ cells (9). The expressions of TXR1 and thrombospondin 1 (TSP1) were significantly correlated with treatment outcome in lung adenocarcinomas (10). A previous study confirmed that TXR1 regulated the cytotoxicity of taxanes in vitro through decreasing the expression of TSP1 in gastric cancer (6). TXR1 regulates not only taxol resistance but also oxaliplatin response; it was reported that overexpression of TXR1 evoked oxaliplatin resistance by regulating TSP1 $(11,12)$. However, the association between TXR1 expression and cisplatin resistance in gastric cancer remains unknown.

In order to clarify the association between TXR1 and cisplatin response in gastric cancer, the expression of TXR1 in cisplatin resistance and sensitive gastric cancer tissues was determined using reverse transcription-quantitative polymerase chain reaction (RT-qPCR), western blot analysis and immunohistochemistry. Next, TXR1 expression was altered in gastric cancer cells by the transfection of lentivirus or small interfering RNA (siRNA), and then cisplatin response was tested using an MTS assay. Animal experiments proved the 
effect of TXR1 in inducing cisplatin resistance in vivo. Further investigation revealed that TXR1 regulated cisplatin resistance via apoptosis. Overall, the results of the present study revealed that TXR1 served a notable function in the response to cisplatin via apoptosis in gastric cancer in vitro and in vivo.

\section{Materials and methods}

Patients and tissues. Tissue specimens were obtained between January 2009 and December 2016. Tissue samples from patients with primary gastric cancer were collected from 18 patients with gastric cancer who received cisplatin-containing chemotherapy and then underwent surgical resection at Beijing Friendship Hospital, Capital Medical University (Beijing, China). Patients were excluded from the study if previous chemotherapy or radiotherapy treatment had been given or if treatment was not successfully completed. A total of 12 males and 6 females were included in those patients, of which the age ranged from 32 to 65 years. The median age of those patients was $55.67 \pm 6.19$ years. Tissues were obtained following chemotherapy treatment. The requirement for informed patient consent was waived by the Ethics Committee of Beijing Friendship Hospital, who approved the present study.

Patients were divided into two groups according to the response to chemotherapy. Patients that exhibited complete or partial response to cisplatin were included in the cisplatin-sensitive category. Patients with progressive disease or stable disease following cisplatin treatment were included in the cisplatin-resistant category. Complete response was defined as complete eradication of all evaluable disease, confirmed by biopsy, for at least 4 weeks. Partial response was defined as a decrease of at least $30 \%$ of total size for at least 4 weeks.

Patients with progressive disease included those that exhibited increase in tumor size and/or worsening of the shape, or novel intragastric lesions. Patients with stable disease were defined as those that exhibited changes in tumor size or shape that were less than a partial response but were not progressive disease.

Cell culture and treatments. The human gastric cancer SGC-7901 cell line was purchased from National Infrastructure of Cell Line Resource (Beijing, China) and stored in of General Surgery Laboratory in the Beijing Friendship Hospital. A cisplatin-resistant subline was established and termed the SGC-7901/DDP cell line. SGC-7901 cells were exposed continuously to $0.01 \mu \mathrm{g} / \mathrm{ml}$ cisplatin at the beginning and the cisplatin concentration was gradually increased in 2 -fold increments $(0.125,0.25,0.5,1,2,4$ and $8 \mu \mathrm{g} / \mathrm{ml})$. Finally, the surviving cells were maintained at $0.1 \mu \mathrm{g} / \mathrm{ml}$ cisplatin. Cell lines were cultured in DMEM supplemented with $10 \%$ fetal bovine serum, $100 \mathrm{U} / \mathrm{ml}$ penicillin and $100 \mu \mathrm{g} / \mathrm{ml}$ streptomycin (Gibco; Thermo Fisher Scientific, Inc., Waltham, MA, USA) and maintained in a $5 \% \mathrm{CO}_{2}$ humidified incubator at $37^{\circ} \mathrm{C}$. Cisplatin was purchased from Qilu Pharmaceutical Co., Ltd. (Jinan, China). Pirarubicin was obtained from Shenzhen Main Luck Pharmaceuticals, Inc. (Shenzhen, China). Fluorouracil (5-FU) was purchased from Shanghai Xudong Haipu Pharmaceutical Co., Ltd. (Shanghai, China). The lentivirus containing TXR1, TXR1-targeting si-TXR1 or negative control for lentivirus and siRNAs were obtained from Shanghai GenePharma Co., Ltd.
Transfection was performed with $100 \mathrm{~nm}$ small interfering RNA (siRNA) using the Lipofectamine ${ }^{\mathrm{TM}}$ RNAiMAX reagent (Invitrogen; Thermo Fisher Scientific, Inc.) according to the manufacturer's protocol. Cells were collected after $48 \mathrm{~h}$ for subsequent experimentations.

The sequences of siRNAs were as follows: si-TXR1 duplex sense, 5'-CAGUGAUAGUAGACAAGAATT-3 and anti-sense, 5'-UUCUUGUCUACUAUCACUGTT-3; and si-NC duplex sense, 5'-UUCUCCGAACCUUCAGUTT-3 and anti-sense, 5'-ACGUGACACGUUCCGAGAATT-3'.

$R T-q P C R$. Total RNA from cell lines and patient tissues was extracted with TRIzol (Invitrogen; Thermo Fisher Scientific, Inc.) in accordance with the manufacturer's protocol. The quality and quantity of RNA were assessed using a NanoDrop ND-1000 spectrophotometer (Thermo Fisher Scientific, Inc., Wilmington, DE, USA). RNA samples were stored at $-80^{\circ} \mathrm{C}$ for subsequent experiments. The Reverse Transcription system (cat. no. A3500; Promega Corporation, Madison, WI, USA) was used to generate cDNA libraries. qPCR was performed with the SYBRGreen mixture (Applied Biosystems; Thermo Fisher Scientific, Inc.) according to the manufacturer's protocol. The PCR thermocycling conditions were as follows: 5 min incubation at $94^{\circ} \mathrm{C}$, followed by 30 cycles of $94^{\circ} \mathrm{C}$ for $30 \mathrm{sec}, 60^{\circ} \mathrm{C}$ for $30 \mathrm{sec}$ and $72^{\circ} \mathrm{C}$ for $30 \mathrm{sec}$, with a final incubation for $5 \mathrm{~min}$ at $72^{\circ} \mathrm{C}$. Relative expression was calculated using $2^{-\Delta \Delta C q}$ method using ABI7500 software (13). $\beta$-actin was used for normalization. All PCR amplifications were performed in triplicate and the experiment was repeated three times. Primers were obtained from Sangon Biotech Co., Ltd (Shanghai, China). The primers used were as follows: TXR1 forward, AAGGTTGCTGGGAAGTAGAGTC and reverse, ATTGGGCTAAGGAGGAGAGGTA; TSP1 forward, CGT GGTCATCTTGTTCTGTGA and reverse, AGGGTTTCC CGTTCATCTG; and $\beta$-actin forward, GCACCACACCTT CTACAATG and reverse, TGCTTGCTGATCCACATCTG.

Western blot analysis. Total protein was extracted from cells using a total protein extraction kit (Nanjing Keygen Biotech Co., Ltd. Nanjing) and quantified using the BCA method (Tiangen Biotech Co., Ltd., Beijing, China) according to the manufacturer's instructions. Protein samples ( $8 \mu \mathrm{g} / \mathrm{lane})$ were separated using $12 \%$ SDS-PAGE and transferred to a polyvinylidene difluoride (PVDF) membrane. PVDF membranes were blocked with $5 \%$ skimmed milk at room temperature for $1 \mathrm{~h}$ and incubated with anti- $\beta$-actin (cat. no. ab6276; 1:3,000) anti-TSP1 (cat. no. ab88529; 1:500; both Abcam, Cambridge, MA, USA) and anti-TXR1 (cat. no. SAB1101786; 1:500; Sigma-Aldrich; Merck KGaA (Darmstadt, Germany) primary antibodies overnight at $4^{\circ} \mathrm{C}$. Membranes were washed 3 times with TBST and subsequently incubated with goat anti-rabbit/goat anti-mouse $\mathrm{IgG}$ secondary antibodies (cat. no. ZB2301/ZB2305; 1:3,000; OriGene Technologies, Inc., Beijing, China) conjugated to horseradish peroxidase at room temperature for $1 \mathrm{~h}$, and peroxidase activity was detected by enhanced chemiluminescence (EMD Millipore, Billerica, MA, USA).

Immunohistochemistry. Formalin-fixed, paraffin-embedded sections (4- $\mu$ m thick) were deparaffinized in xylene, rehydrated 
in a graded ethanol series $(100,95,90,80$ and $70 \%)$ and treated with $3 \%$ hydrogen peroxide solution at room temperature for $10 \mathrm{~min}$. Epitope retrieval was performed in boiling water $\left(>95^{\circ} \mathrm{C}\right.$ ) with $0.01 \mathrm{M}$ citric acid sodium buffer solution ( $\mathrm{pH}$ 6.0) for $40 \mathrm{~min}$. The sections were blocked with $5 \%$ goat serum at room temperature for $20 \mathrm{~min}$ and incubated with TXR1 antibodies (cat. no. HPA046219; 1:100; Sigma-Aldrich; Merck $\mathrm{KGaA}$ ) overnight at $4^{\circ} \mathrm{C}$. Next, goat anti-rabbit IgG conjugated to biotin (cat. no. TA130017; 1:200; OriGene Technologies, Inc., Beijing, China) was added to the slices, and incubated at $37^{\circ} \mathrm{C}$ for $30 \mathrm{~min}$. Subsequently, detection was performed by 3,3'-diaminobenzidine (DAB) chromogenic reaction. Staining results were visualized using an Olympus CX31-LV320 light microscope (Olympus Corporation, Tokyo, Japan) at a magnification of $\mathrm{x} 200$.

MTS assay. Drug response in gastric cancer cells was analyzed in triplicate using the CellTiter 96 AQueous One Solution Cell Proliferation assay (MTS assay; Promega Corporation) in accordance with the manufacturer's protocols. In total, 3,000 cells/well were seeded in 96-well plates and exposed to various concentrations of drugs (cisplatin ranging from 0.125 to $8 \mu \mathrm{g} / \mathrm{ml}$ in 2 -fold increments; pirarubicin ranging from 0.25 to $16 \mu \mathrm{g} / \mathrm{ml}$ in 2-fold increment and 5-FU ranging from 0.5 to $32 \mu \mathrm{g} / \mathrm{ml}$ in 2-fold increments). After $48 \mathrm{~h}$ of treatment, $20 \mu \mathrm{l}$ MTS was added to the medium and the cells were incubated in a $5 \% \mathrm{CO}_{2}$ incubator at $37^{\circ} \mathrm{C}$ for $2-4 \mathrm{~h}$. The absorbance was measured at $490 \mathrm{~nm}$ using a plate reader.

Apoptosis analysis. Caspase-3/7 activity was tested using the Caspase-Glo3/7-assay (Promega Corporation) according with the manufacturer's protocol. In total, 3,000 cells/well were planted into 96-well plate and treated with cisplatin at the dosage of $0.25,0.5,1,2$ or $4 \mu \mathrm{g} / \mathrm{ml}$ for $48 \mathrm{~h}$, then Caspase- $\mathrm{Glo} 3 / 7^{\circledR}$ reagent was added and luminescence was recorded by Fluostar Optima plate reader (BMG Labtechnologies $\mathrm{GmbH}$, Inc., Durham, NC, USA) at $37^{\circ} \mathrm{C}$ and the enzymatic activity was calculated.

Xenograft mouse model. A total of 20 male BALB/c nude mice (4-6 weeks old, 18-22 g) were purchased from Beijing Vital River Laboratory Animal Technology Co., Ltd. (Beijing, China) and kept in the Animal Laboratory of Beijing Friendship Hospital for experimentation. Mice were maintained under a $6 / 18 \mathrm{~h}$ light-dark cycle at $22 \pm 1^{\circ} \mathrm{C}$. The mice had ad libitum access to tap water and food. Care was taken to avoid environmental stress prior to and during the course of the experiments (including noise, smell or cage crowding). Animal studies were performed in conformity with applicable laws and guidelines and were approved by the Laboratory Animal Center at Beijing Friendship Hospital. $1 \times 10^{6}$ cells were inoculated subcutaneously into the armpit of right forelimb of the mice to generate the xenograft model. When the tumor volume reached $100 \mathrm{~mm}^{3}$, the mice were given the appropriate treatment. In total, 20 mice were divided into 4 groups: i) The SGC-7901/DDP NC group, consisting of mice injected with $1 \times 10^{6}$ SGC-7901/DDP cells and intraperitoneally treated with physiological saline twice a week for 4 weeks; ii) SGC-7901/DDP cisplatin group, consisting of mice injected with $1 \times 10^{6}$ SGC-7901/DDP cells and intraperitoneally treated with $2.5 \mathrm{mg} / \mathrm{kg}$ cisplatin twice a week for 4 weeks; iii) the
SGC-7901/DDP/si-TXR1 group, consisting of mice injected with $1 \times 10^{6}$ SGC-7901/DDP cells transfected with lentivirus to knockdown expression of TXR1 and injected into mice subcutaneously. Then mice were treated intraperitoneally with $2.5 \mathrm{mg} / \mathrm{kg}$ cisplatin twice a week for 4 weeks; and iv) the SGC-7901/DDP lentivirus group, consisting of mice injected with $1 \times 10^{6}$ SGC-7901/DDP cells that were treated with lentivirus that interfered with TXR1 expression intratumorally for 4 weeks, $10^{7}$ transduction units per mouse, twice a week. Tumor size was measured twice per week. Tumor volume was calculated as follows: Volume=length/width $2 / 2$.

Statistical analysis. The results are presented as the mean \pm standard deviation. All data were analyzed using SPSS 17.0 software (SPSS, Inc., Chicago, IL, USA). Significant differences were assessed using a standard one-way analysis of variance followed by Dunnett's multiple comparisons test or Student's t-test. The Kolmogorov-Smirnov test for normality was applied to assess normal distribution. $\mathrm{P}<0.05$ was considered to indicate a statistically significant difference.

\section{Results}

Association between TXR1 level expression level and cisplatin response in gastric cancer tissues. The present study included 18 patients with gastric cancer who were treated with cisplatin-based chemotherapy at Beijing Friendship Hospital. Patients were divided into two groups according to the response to chemotherapy and the expression of TXR1 was assessed in these two groups. Results revealed that TXR1 expression was significantly elevated in the cisplatin-resistant group (Fig. 1A), which indicated that TXR1 might be a regulator of cisplatin resistance. Representative TXR1 expression in cisplatin-resistant and-sensitive tissues was assessed using western blot and immunohistochemistry, depicted in Fig. $1 \mathrm{~B}$ and $\mathrm{C}$, respectively.

Establishment a cisplatin-resistant sublines with SGC-7901 cell line. To investigate the association between TXR1 expression and cisplatin-resistance in gastric cancer cell lines, a cisplatin-resistant gastric cancer cell sublines was established. This subline proved $\sim 6$-fold resistant to cisplatin (Fig. 2A and B). This level of resistance was confirmed to be stable over 3 months in continuous culture.

Dose-response data for sensitive cells and resistant sublines demonstrated that cisplatin-resistant cells were cross-resistant to 5-FU and pirarubicin (Fig. 2C and D).

Cisplatin sensitivity of gastric cancer cells is associated with endogenous TXR1 expression. The expression of TXR1 was assessed in cisplatin-resistant and -sensitive cell lines. Results revealed that TXR1 was significantly increased in cisplatin-resistant cells (Fig. 3A), indicating that TXR1 has a role in regulating cisplatin response.

To investigate the role of TXR1 in cisplatin resistance, TXR1 lentivirus (or a control equivalent) was transfected into SGC-7901 cells, which significantly increased the TXR1 expression level (Fig. 3B). Next, the SGC-7901 cells transfected with control or TXR1 lentivirus were 
A

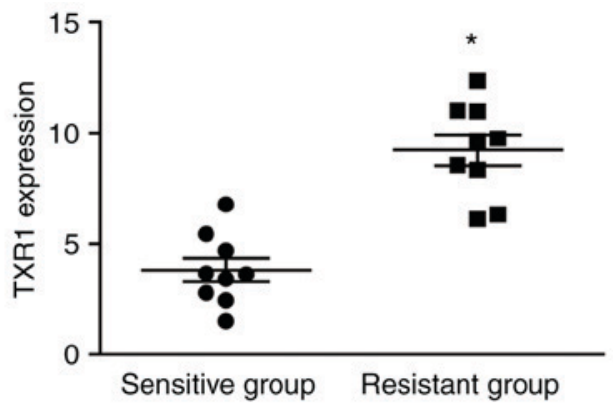

C

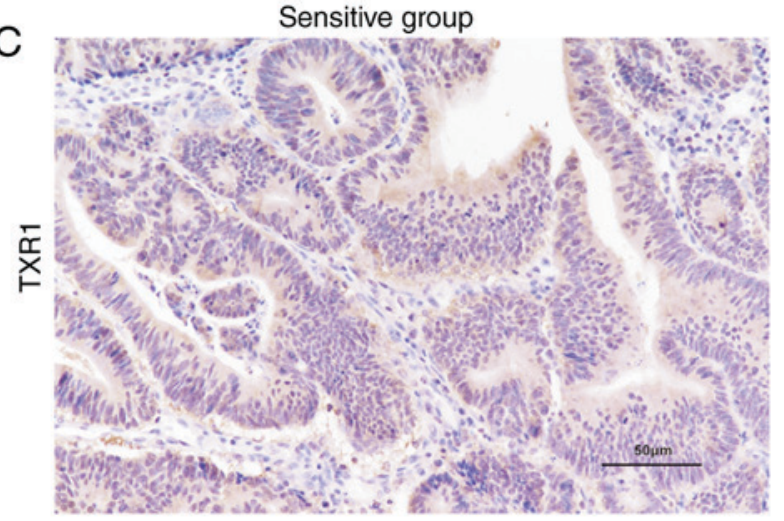

B

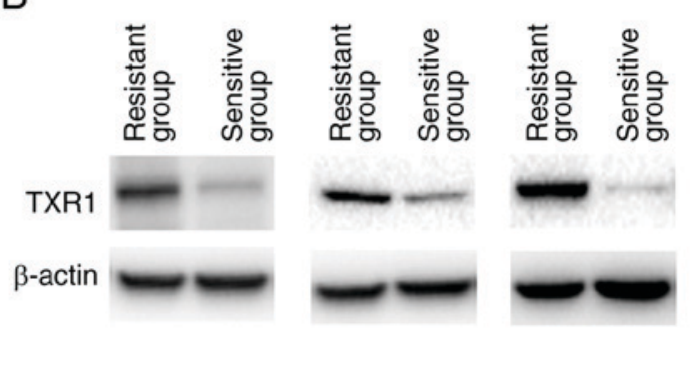

Resistant group

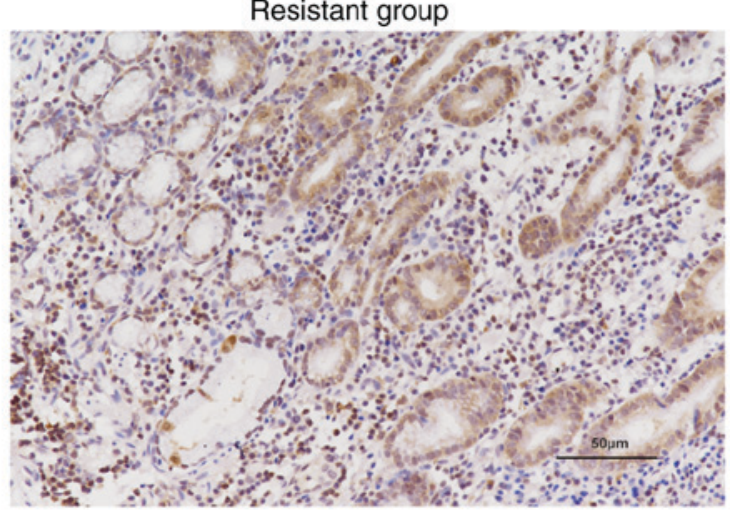

Figure 1. Association between the level of TXR1 expression and cisplatin response in gastric cancer tissues. (A) The expression of TXR1 in fresh-frozen tissues of gastric cancer patients who were sensitive or resistance to cisplatin was detected by reverse transcription-quantitative polymerase chain reaction. $\beta$-actin was used for normalization. "P<0.05 vs. sensitive group. Error bars, mean \pm standard deviation. (B) Representative TXR1 expression in cisplatin-resistant and cisplatin-sensitive tissues was observed by western blot analysis. $\beta$-actin was used for normalization. (C) Representative TXR1 expression in cisplatin-resistant and cisplatin-sensitive tissues, depicted using immunohistochemistry. Scale bars, $50 \mu \mathrm{m}$. TXR1, taxol resistance protein 1.
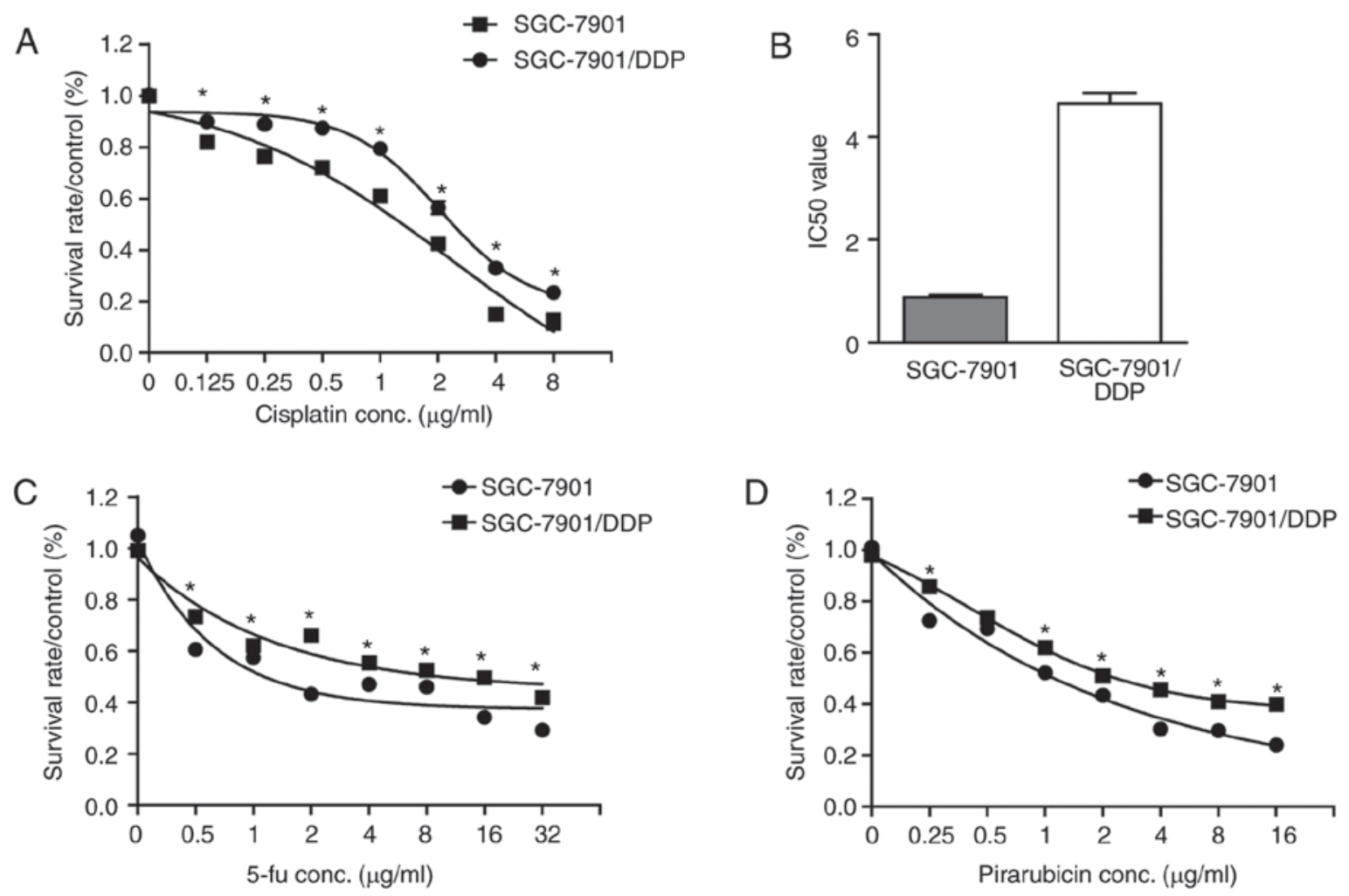

Figure 2. Establishment of a cisplatin-resistant SGC-7901 subline. (A) SGC-7901 cells were exposed to cisplatin continually to establish a cisplatin-resistant cell line (the SGC-7901/DDP cell line). Cisplatin sensitivity of the SGC-7901/DDP cell line and its parental cell line were assessed by MTS assay. "P<0.05 vs. SGC-7901. (B) Half-maximal inhibitory concentration of SGC-7901/DDP cells and SGC-7901 cells were calculated. (C) Chemosensitivity of the SGC-7901/DDP and SGC-7901 cell lines to 5-FU were assessed using an MTS assay. "P<0.05 vs. SGC-7901. (D) Chemosensitivity of the SGC-7901/DDP and SGC-7901 cell lines to pirarubicin were assessed by MTS assay. "P<0.05 vs. SGC-7901. Each point is the mean value for three independent experiments; error bars, mean \pm standard deviation. 5-FU, fluorouracil. 
A

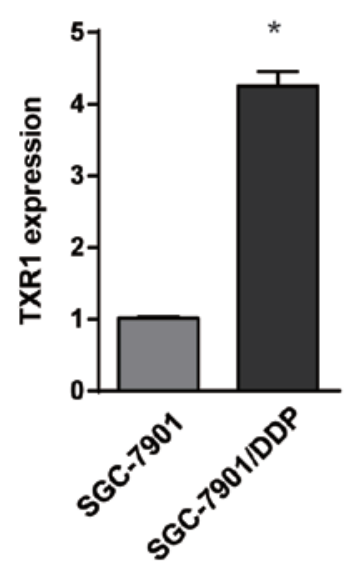

B

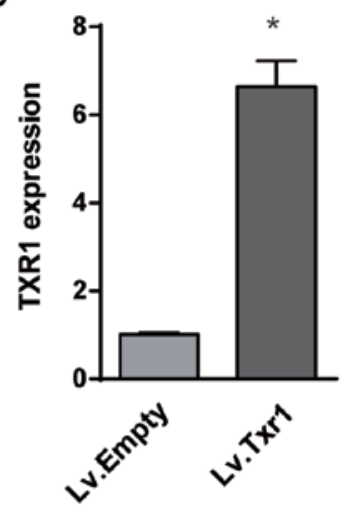

C

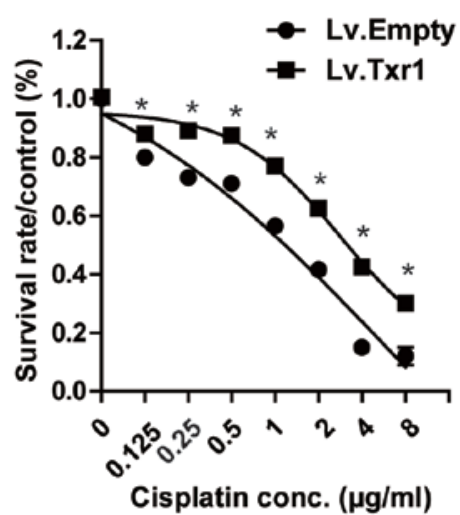

D

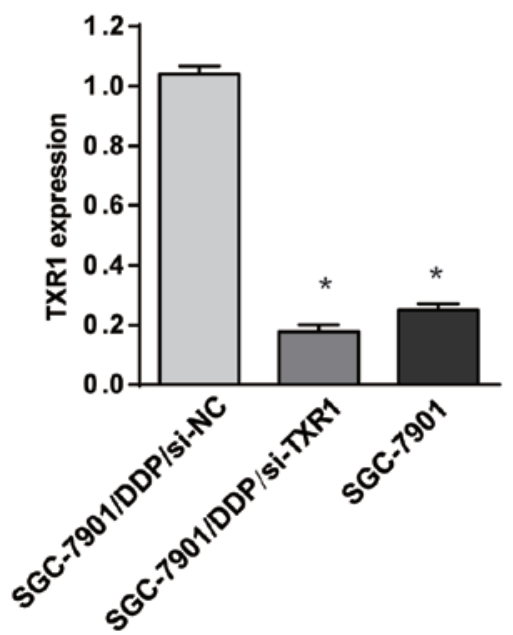

E

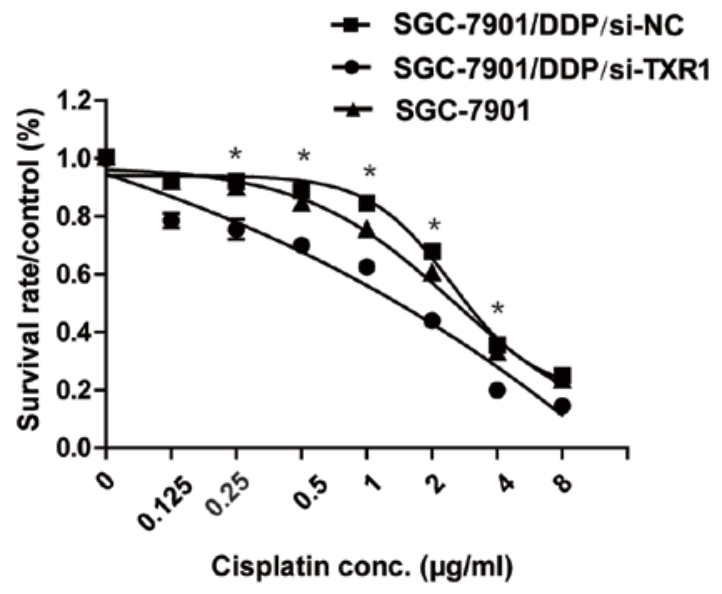

Figure 3. Cisplatin sensitivity of GC cells is associated with endogenous TXR1 expression. (A) The expression of TXR1 in SGC-7901/DDP cell line and its parental cell were tested by RT-qPCR. $\beta$-actin was used for normalization. * P<0.05, compared with SGC-7901. (B and C) Lv.TXR1 or Lv.NC were transfected into SGC-7901 cells. (B) TXR1 level was confirmed by RT-qPCR and (C) cisplatin sensitivity were determined by MTS assays. $\beta$-actin was used for normalization. ${ }^{*} \mathrm{P}<0.05$, compared with Lv.Empty. (D and E) si-NC or si-TXR1 were transfected into SGC-7901/DDP cells. (D) TXR1 level was confirmed by RT-qPCR and (E) cisplatin sensitivity were tested by MTS assay. $\beta$-actin was used for normalization. *P<0.05, compared with SGC-7901/DDP/si-NC. Points, mean values for three independent experiments; error bars, mean \pm standard. GC, gastric cancer; TXR1, taxol resistance protein 1; RT-qPCR, reverse transcription-quantitative polymerase chain reaction; Lv.TXR1, lentivirus bearing TXR1; NC, negative control; si-NC, NC short interfering RNA.

treated with cisplatin at various concentrations. The results revealed that TXR1 overexpression significantly increased the resistance of cisplatin (Fig. 3C). Next, SGC-7901/DDP cells were transfected with siRNA to knock-down TXR1 (Fig. 3D). The resistance to cisplatin was significantly decreased in SGC-7901/DDP/si-TXR1 cells compared with SGC-7901/DDP/si-NC cells $(\mathrm{P}<0.05$; Fig. 3E). Similarly, the resistance of cisplatin was decreased in SGC-7901 cells compared with SGC-7901/DDP/si-NC cells, where the TXR1 level was lower compared with SGC-7901 cells (Fig. 3E). In summary, the results of the present study indicated a notable role of TXR1 in cisplatin response in gastric cancer.

TXR1 enhance the cisplatin resistance in gastric cancer in vivo. To investigate the effect of TXR1 in cisplatin resistance in vivo, a xenograft model was established in BALB/c nude male mice.

As shown in Fig. 4A and B, the difference in tumor volumes in the SGC-7901/DDP NC and SGC-7901/DDP cisplatin groups was not significant, owing to the cisplatin resistance of SGC-7901/DDP cells. Compared with the SGC-7901/DDP cisplatin group, the tumor volume in the SGC-7901/DDP-siTXR1 group was significantly smaller, indicating that TXR1 has a notable role in cisplatin response in vivo. Similarly, lentivirus was injected into tumor to silence TXR1 expression, generating similar data to the SGC-7901/DDP/si-TXR1 group, which further confirmed these results.

TXR1 enhance the cisplatin resistance through inducing apoptosis. Owing to the inverse association observed between TXR1 levels and cisplatin sensitivity, additional mechanistic experiments were warranted. As apoptosis is the predominant mechanism of cisplatin-induced toxicity, an apoptosis assay was performed using a caspase-glo3/7 assay. The results of this assay revealed that TXR1-knockdown significantly increased the cisplatin-induced activity of caspase-3/7, whereas TXR1-overexpression reduced the activity of caspase- $3 / 7$ in 
A

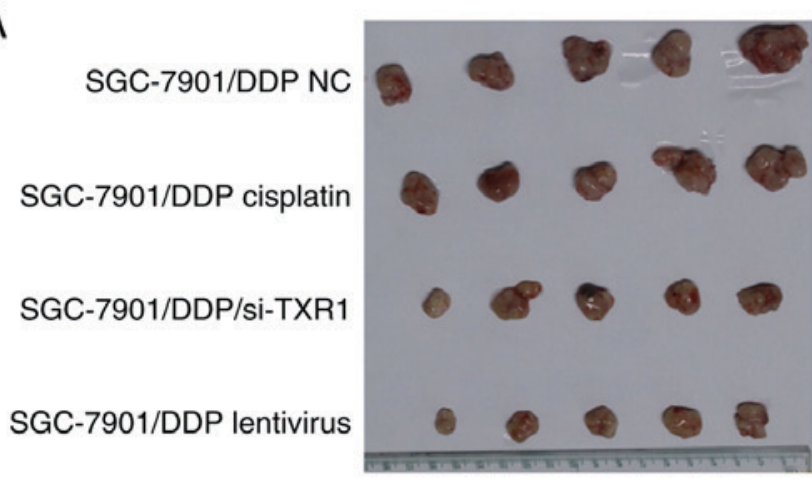

B

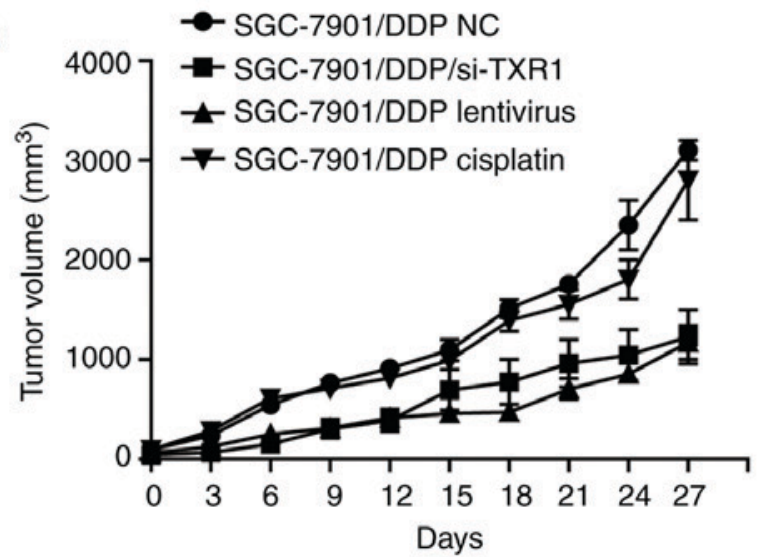

Figure 4. TXR1 enhances cisplatin resistance in gastric cancer in vivo. (A) Mice were inoculated subcutaneously with $1 \times 10^{6}$ cells in the right flank. Representative images were captured at the end of 27 days. (B) Tumor volume was measured at each time point. Data are presented as the mean \pm standard deviation ( $\mathrm{n}=5$ per group). TXR1, taxol resistance protein 1 .

A

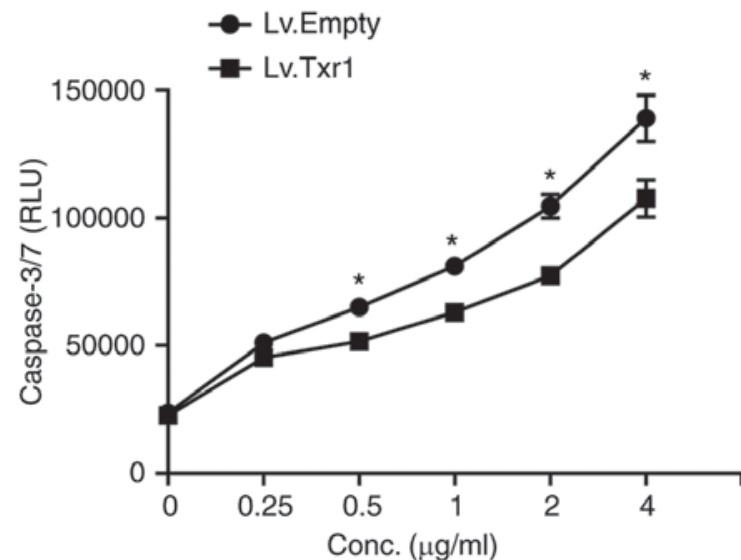

C

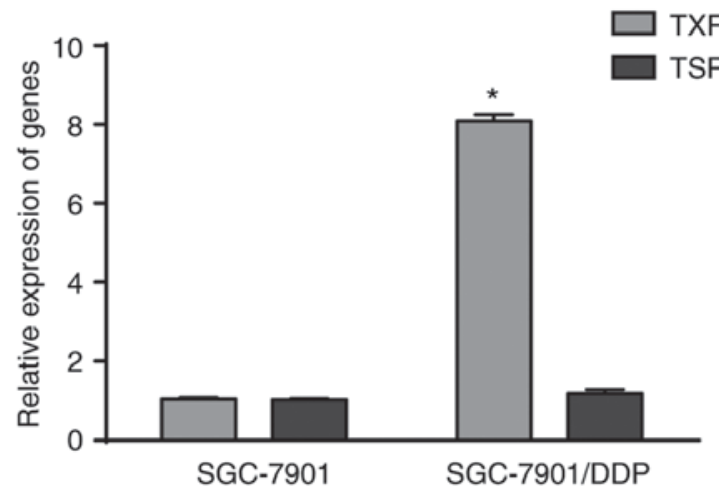

B

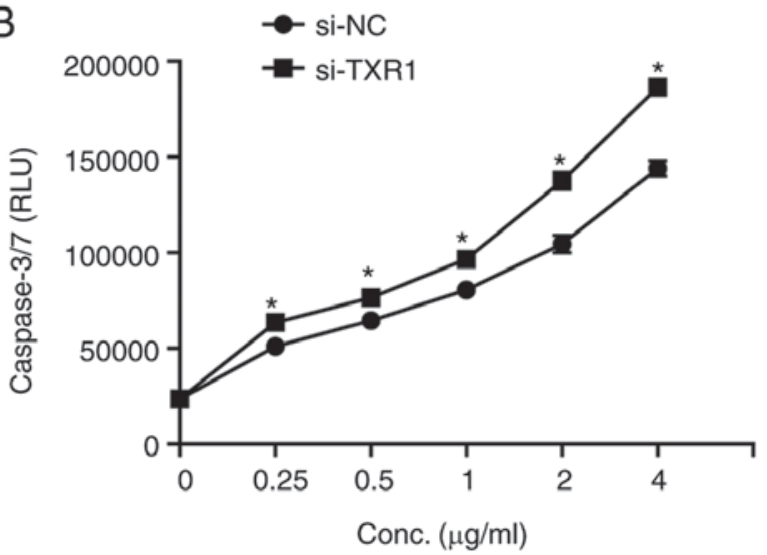

D

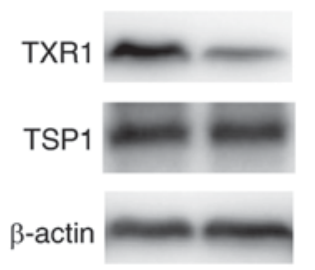

Figure 5. TXR1 enhances cisplatin resistance by inhibiting apoptosis. (A) Lv.TXR1 was transfected into SGC-7901 cells to overexpress TXR1, then the caspase3/7 activity was assessed using the Caspase-Glo3/7-assay. Each point represents the mean RLU \pm SEM of triplicate samples from two independent experiments. "P<0.05, vs. Lv.Empty. (B) SGC-7901 cells were transfected with si-TXR1 to reduce the expression of TXR1, then caspase-3/7 activity was assessed using the Caspase-Glo3/7-assay. Points on graph represent the mean RLU \pm SEM of triplicate samples from two independent experiments. "P<0.05 vs. si-NC. (C and D) the level of TXR1 and TSP1 in SGC-7901/DDP cell line and its parental cell were detected by (C) reverse transcription-quantitative polymerase chain reaction and (D) western blot. $\beta$-actin was used for normalization. "P $<0.05$, compared with SGC-7901. Points, mean values for three independent experiments. Error bars, \pm SEM. TXR1, taxol resistance protein 1; Lv.TXR1, lentivirus bearing TXR1; RLU, relative luminescence units; SD, standard deviation; si-TXR1, small interfering RNA targeting TXR1.

SGC-7901 cells (Fig. 5A and B). These data indicated that TXR1 expression enhances the cytotoxic effect of cisplatin by sensitizing the gastric cancer cells to cisplatin-induced apoptosis.

The TXR1/TSP1 regulatory pathway has been recently described to be involved in drug resistance in cancer $(6,14)$. Therefore, the expression of TSP1 in SGC-7901 and
SGC-7901/DDP cells was examined at the transcriptional and translational level using RT-qPCR and western blot analysis. Results revealed that there were no significant differences TSP1 at either the mRNA or protein level in SGC-7901/DDP cells (Fig. 5C and D). These results indicated that TSP1 may not be involved in the cisplatin response regulated by TXR1. 


\section{Discussion}

Due to a lack of effective screening methods, a majority of patients with gastric cancer are diagnosed at an advanced disease stage when the tumor is inoperable; and for these patients, systemic chemotherapy is the main treatment option $(15,16)$. Cisplatin is the basis of multiple treatment regimens; it exerts its cytotoxicity activity mainly in combination with other chemotherapy drugs by inducing apoptosis in cancer cells (4). However, with multiple drug treatments, cancer cells frequently acquire resistance to the cytotoxic effects of cisplatin (17). The problems caused by cisplatin resistance seem to be more severe than they were in the past (18).

The mechanism of drug resistance contains a series of pathological changes induced by expression diversification of large-scale genes in numerous cell lines (19-21). Recent studies showed that TXR1 was a taxol-resistance-associated agent, which also regulates oxaliplatin response in gastric cancer $(4,6)$. TXR1 was previously shown to be closely associated with oxaliplatin response in vitro. However, to the best of our knowledge, the association between TXR1 and cisplatin resistance in vitro and in vivo was unknown. It was inferred that TXR1 may have a role in cisplatin resistance. The differential expression of TXR1 between cisplatin-resistant and cisplatin-sensitive gastric cancer tissues provided clues for this hypothesis. Subsequently, the SGC-7901/DDP cell line was established. Compared with its parental SGC-7901 cell line, TXR1 expression was elevated in the SGC-7901/DDP cell line. Overexpression of TXR1 led to the development of cisplatin resistance in SGC-7901 cell lines, whereas downregulation of TXR1 reversed the resistance induced by TXR1 in the SGC-7901/DDP cell line. All results indicated that TXR1 may protect gastric cancer cells from cisplatin cytotoxicity in the gastric cancer SGC-7901 cell line. The results of the in vivo experiment further confirmed that TXR1 was likely associated with cisplatin resistance. In the present study, TXR1 exerted effects as an inducer of cisplatin resistance and could be considered to be cancer-promoting gene. This result is consistent with previous research, in which TXR1 expression caused multiple drug resistance $(8,22)$. Downregulation of the apoptotic signal is a common characteristic of multi-drug resistance (23). Although cisplatin is a potent inducer of apoptosis, resistance is considered to be developed when tumor cells fail to undergo apoptosis at clinical drug concentrations $(24,25)$. Thus, the present study assessed the apoptosis rate induced by changes to TXR1 expression. Caspase-3/7 have been reported to be activated by chemotherapy-induced tumor cell death and considered to be an early biomarker for evaluating apoptosis-inducing antitumor effects (26). The activity of Caspase-3/7 was detected to evaluate the rate of apoptosis induced by cisplatin. Results revealed that the upregulation of TXR1 caused an increase in the apoptotic rate, whereas downregulation of TXR1 caused a decrease. Thus, we hypothesize that TXR1 induces cisplatin resistance by regulating apoptosis.

It has been reported that the TXR1 protein impedes taxane-induced apoptosis via downregulation of thrombospondin 1 (TSP1) (14,27). TSP1 has been suggested to serve a notable role in preventing angiogenesis and inducing apoptosis in malignant cells (28). Thus, the present study also analyzed TSP1 gene expression. Unexpectedly, changes in the expression of TSP1 were not observed in cisplatin-resistant cells. Therefore, TSP1 was not involved in cisplatin response in gastric cancer. The specific molecular mechanism of cisplatin resistance induced by TXR1 requires further investigation.

In conclusion, to the best of our knowledge, the present study reveals, to the best of our knowledge for the first time, that TXR1-knockdown enhanced chemosensitivity to cisplatin in gastric cancer cells by inducing apoptosis. The identification of TXR1 as an inducer of chemoresistance in patients undergoing cisplatintreatment providesa theoretical basis for novelapproaches to overcoming chemotherapy resistance.

\section{Acknowledgements}

Not applicable.

\section{Funding}

The present study was supported by the grants from National Natural Science Foundation of China (no. 81172317 to Zhigang Bai).

\section{Availability of data and materials}

The datasets used and/or analyzed during the current study are available from the corresponding author on reasonable request.

\section{Authors' contributions}

ZZand ZB participated in the design of the study and performed the majority of the analyses. SD drafted the manuscript. SD and JY conceived and coordinated the study. All authors have read and approved the final manuscript.

\section{Ethics approval and consent to participate}

Ethical approval was provided by the Ethics Committee of Beijing Friendship Hospital (reference no. BJFH-EC/2014-051), and the requirement for informed patient consent was waived by the Ethics Committee of Beijing Friendship Hospital.

\section{Consent for publication}

The present study was granted an exemption by the Ethics Committee of Beijing Friendship Hospital as the patients cannot be traced.

\section{Competing interests}

The authors declare that they have no competing interests.

\section{References}

1. Kato M and Asaka M: Recent knowledge of the relationship between Helicobacter pylori and gastric cancer and recent progress of gastroendoscopic diagnosis and treatment for gastric cancer. Jpn J Clin Oncol 40: 828-837, 2010.

2. Ikeda F and Kiyohasa Y: The epidemiology of gastric cancer: The Hisayama Study. Fukuoka Igaku Zasshi 106: 195-201, 2015 (In Japanese). 
3. De Milito A and Fais S: Tumor acidity, chemoresistance and proton pump inhibitors. Future Oncol 1: 779-786, 2005.

4. Florea AM and Büsselberg D: Cisplatin as an anti-tumor drug: Cellular mechanisms of activity, drug resistance and induced side effects. Cancers (Basel) 3: 1351-1371, 2011.

5. Siddik ZH: Cisplatin: Mode of cytotoxic action and molecular basis of resistance. Oncogene 22: 7265-7279, 2003.

6. Bai ZG, Qu X, Han W, Ma XM, Zhao XM and Zhang ZT: Expression of taxol resistance gene 1 correlates with gastric cancer patient clinical outcome and induces taxol resistance. Mol Med Rep 3: 1071-1078, 2010.

7. Zhang H, Qu X, Ma X, Wang T, Yang Y, Ge Z, Zhang Z, Bai Z, Gao Y, Yuan Z and Wang Z: TXR1 and TSP1 expression varies by the molecular subtypes of breast cancer patients who received previous docetaxel-based first-line chemotherapy. Exp Biol Med (Maywood) 241: 1919-1923, 2016.

8. Papadaki C, Tsaroucha E, Kaklamanis L, Lagoudaki E, Trypaki M, Tryfonidis K, Mavroudis D, Stathopoulos E, Georgoulias V and Souglakos J: Correlation of BRCA1, TXR1 and TSP1 mRNA expression with treatment outcome to docetaxel-based first-line chemotherapy in patients with advanced/metastatic non-small-cell lung cancer. Br J Cancer 104 316-323, 2011.

9. Bi W, Wang Y, Sun G, Zhang X, Wei Y, Li L and Wang X: Paclitaxel-resistant HeLa cells have up-regulated levels of reactive oxygen species and increased expression of taxol resistance gene 1. Pak J Pharm Sci 27: 871-878, 2014.

10. Papadaki C, Mavroudis D, Trypaki M, Koutsopoulos A Stathopoulos E, Hatzidaki D, Tsakalaki E, Georgoulias V and Souglakos J: Tumoral expression of TXR1 and TSP1 predicts overall survival of patients with lung adenocarcinoma treated with first-line docetaxel-gemcitabine regimen. Clin Cancer Res 15: 3827-3833, 2009.

11. Bi J, Bai Z, Ma X, Song J, Guo Y, Zhao J, Yi X, Han S and Zhang Z: Txr1: An important factor in oxaliplatin resistance in gastric cancer. Med Oncol 31: 807, 2014.

12. Liu L, Bai Z, Ma X, Wang T, Yang Y and Zhang Z: Effects of taxol resistance gene 1 expression on the chemosensitivity of SGC-7901 cells to oxaliplatin. Exp Ther Med 11: 846-852, 2016.

13. Livak KJ and Schmittgen TD: Analysis of relative gene expression data using real-time quantitative PCR and the 2(-Delta Delta C(T)) method. Methods 25: 402-408, 2001.

14. van Amerongen R and Berns A: TXR1-mediated thrombospondin repression: A novel mechanism of resistance to taxanes? Genes Dev 20: 1975-1981, 2006.

15. Songun I, Keizer HJ, Hermans J, Klementschitsch P, de Vries JE, Wils JA, van der Bijl J, van Krieken JH and van de Velde CJ: Chemotherapy for operable gastric cancer: Results of the Dutch randomised FAMTX trial. The Dutch Gastric Cancer Group (DGCG). Eur J Cancer 35: 558-562, 1999.

16. Wagner AD, Grothe W, Haerting J, Kleber G, Grothey A and Fleig WE: Chemotherapy in advanced gastric cancer: A systematic review and meta-analysis based on aggregate data. J Clin Oncol 24: 2903-2909, 2006.
17. Tsuruo T, Naito M, Tomida A, Fujita N, Mashima T, Sakamoto H and Haga N: Molecular targeting therapy of cancer: Drug resistance, apoptosis and survival signal. Cancer Sci 94: 15-21, 2003.

18. Galluzzi L, Senovilla L, Vitale I, Michels J, Martins I, Kepp O, Castedo M and Kroemer G: Molecular mechanisms of cisplatin resistance. Oncogene 31: 1869-1883, 2012.

19. Housman G, Byler S, Heerboth S, Lapinska K, Longacre M, Snyder N and Sarkar S: Drug resistance in cancer: An overview. Cancers (Basel) 6: 1769-1792, 2014.

20. Rueff J and Rodrigues AS: Cancer drug resistance: A brief overview from a genetic viewpoint. Methods Mol Biol 1395: 1-18, 2016.

21. Wu Q, Yang Z, Nie Y, Shi Y and Fan D: Multi-drug resistance in cancer chemotherapeutics: Mechanisms and lab approaches. Cancer Lett 347: 159-166, 2014.

22. Colloca G, Venturino A and Checcaglini F: Second-line chemotherapy in metastatic docetaxel-resistant prostate cancer: A review. Med Oncol 29: 776-785, 2012.

23. Holohan C, Van Schaeybroeck S, Longley DB and Johnston PG: Cancer drug resistance: An evolving paradigm. Nat Rev Cancer 13: 714-726, 2013.

24. O'Neill CF, Ormerod MG, Robertson D, Titley JC, Cumber-Walsweer Y and Kelland LR: Apoptotic and non-apoptotic cell death induced by cis and trans analogues of a novel ammine(cyclohexylamine)dihydroxodichloroplatinum(IV) complex. Br J Cancer 74: 1037-1045, 1996.

25. Henkels KM and Turchi JJ: Induction of apoptosis in cisplatin-sensitive and -resistant human ovarian cancer cell lines. Cancer Res 57: 4488-4492, 1997.

26. Ye D, Shuhendler AJ, Pandit P, Brewer KD, Tee SS, Cui L, Tikhomirov G, Rutt B and Rao J: Caspase-responsive smart gadolinium-based contrast agent for magnetic resonance imaging of drug-induced apoptosis. Chem Sci 4: 3845-3852, 2014.

27. Lih CJ, Wei W and Cohen SN: Txr1: A transcriptional regulator of thrombospondin-1 that modulates cellular sensitivity to taxanes. Genes Dev 20: 2082-2095, 2006.

28. Sid B, Sartelet H, Bellon G, El Btaouri H, Rath G, Delorme N, Haye B and Martiny L: Thrombospondin 1: A multifunctional protein implicated in the regulation of tumor growth. Crit Rev Oncol Hematol 49: 245-258, 2004.

This work is licensed under a Creative Commons Attribution-NonCommercial-NoDerivatives 4.0 International (CC BY-NC-ND 4.0) License. 